\title{
Sex, age and size as factors affecting the length of stay of dogs in Czech shelters
}

\author{
Jiř́ Žák, Eva Voslářová, Vladimír Večerek, Iveta Bedáňová \\ University of Veterinary and Pharmaceutical Sciences Brno, Faculty of Veterinary Hygiene and Ecology, \\ Department of Veterinary Public Health and Animal Welfare, Brno, Czech Republic
}

Received January 29, 2015
Accepted November 10, 2015

\begin{abstract}
Certain factors associated with the characteristics of sheltered dogs can be important in the adoption process. This study focused on the sex, age, and size of abandoned dogs in Czech shelters. Abandoned male dogs remained significantly longer in shelters (median 27 days) compared to abandoned females (median 21 days). With respect to potential adopters, bitches were the preferred sex in Czech shelters. Young abandoned dogs up to the age of one year had the shortest length of stay (median 19 days), whereas older dogs had the longest length of stay, i.e. dogs in the age range of 7-9 years (median 53.5 days) as well as dogs older than 9 years (median 54 days). Abandoned dogs over $65 \mathrm{~cm}$ at the withers, i.e. giant dogs, and abandoned dogs up to $35 \mathrm{~cm}$ at the withers, i.e. small dogs, had the shortest length of stay in Czech shelters, with medians of 16 days and 21 days, respectively. Medium-sized dogs, i.e. dogs measuring between $35-50 \mathrm{~cm}$ at the withers, and large dogs, i.e. dogs measuring between $51-65 \mathrm{~cm}$ at the withers had the longest length of stay in shelters. A better understanding of factors that negatively affect the adoption process in abandoned sheltered dogs can significantly shorten the length of stay of such dogs in Czech shelters and help to improve the adoption process itself as well as the welfare of sheltered dogs.
\end{abstract}

Time to adoption, sheltered dog, stray, welfare, rehoming

Stray and abandoned animals are a potential source of danger to public health, the environment, and other animals. The medical histories of stray or abandoned animals are often unknown and such dogs may be potential sources of infections that are dangerous to both humans and animals. The issue of stray and abandoned companion animals also pertains to veterinary health care and the question of animal protection and welfare. Companion animals admitted to shelters usually fall into three categories: stray or lost companion animals caught by municipal dog catchers, stray animals brought to the shelter by their finders, or companion animals relinquished by their original owners (Notaro 2004). In some countries the law forbids euthanizing shelter dogs unless they are severely ill. In these cases, dogs could remain in shelters for extended periods of time until they are adopted (Normando et al. 2006). Some authors reported dogs remaining at the shelter for over 1 year (Brown et al. 2013) or even over 5 years (Wells et al. 2002).

Dog's morphology is important in predicting adoption (Protopopova et al. 2012; Voslářová et al. 2015), as are some other characteristics. Size (Brown et al. 2013), sex (Patronek et al. 1995; Lepper et al. 2002; Clevenger and Kass 2003; Diesel et al. 2007), and age (Hart et al. 1998; Lepper et al. 2002; Clevenger and Kass 2003; Němcová and Novák 2003; Brown et al. 2013) have been reported to influence adoption success. These characteristics may vary regionally and depend on the preferences of potential adopters (Normando et al. 2006; Brown et al. 2013) and temporal fluctuations in the availability of animals (Patronek et al. 1995; Normando et al. 2006). Significant differences between shelters regarding the admission characteristics of the dogs, length of stay, and outcomes were observed and it is important to characterize these differences

Address for correspondence:

MVDr. Jiří Žák

Department of Veterinary Public Health and Animal Welfare

Faculty of Veterinary Hygiene and Ecology

University of Veterinary and Pharmaceutical Sciences Brno

Palackého tř. 1/3, 61242 Brno, Czech Republic

Phone: +420 541562778

E-mail: zakj@vfu.cz

http://actavet.vfu.cz/ 
so that government policy can be informed by factors affecting shelter demographics (Marston et al. 2005). Furthermore, understanding the traits of dogs in a specific shelter and the characteristics of these nonhuman animals desired by adopters are critical to improving the welfare of animals served by that shelter (Brown et al. 2013).

The aim of this study was to assess the numbers of incoming and outgoing dogs in selected shelters in the Czech Republic over a period of four years (2010-2013), dividing the outgoing dogs into those reclaimed by their original owner and those adopted by new owners, and sorting them further according to sex, age, and size in order to assess the influence of the selected factors on the length of stay in Czech shelters. Our expectation was to identify some important factors in relation to dogs that can influence the length of stay (LOS) in Czech shelters, with the intention to help to improve the adoption process itself as well as the welfare of dogs in Czech shelters.

\section{Materials and Methods}

Records on sheltered dogs were collected from three municipal dog shelters situated in different regions of the Czech Republic. These shelters are situated in the towns of Havírov, Jihlava, and Kolín. The subjects of this retrospective study were all impounded dogs housed by the shelters from 1 January 2010 to 31 December 2013. For the purposes of this study, dogs were divided into two groups. One group was comprised of dogs that were reclaimed from the shelters by their owners (lost dogs). The second group was comprised of dogs that were abandoned and subsequently adopted. Dogs that were neither reclaimed nor rehomed within the monitored period and remained in the shelters after 31 December 2013 were not included in the analysis. Sex, age and size were monitored in all dogs in both groups. The length of stay (LOS) of each dog was examined to determine how sex, age, and size influenced the probability of the dog being adopted or reclaimed by its owner and the duration of time spent in the shelter. The length of stay was defined as the number of days from the intake date to the date when the dog was adopted or returned to its owner.

The results were analysed using the statistical package Unistat 5.6. (Unistat Ltd., London, UK). Four independent variables were constructed from the shelter's original information: reason for admission ( 2 levels: abandoned, lost), sex (2 levels: male, female), age (5 levels: < 1 year, 1-3 years, 4-6 years, 7-9 years, >9 years), and size at the withers (4 levels: $<35 \mathrm{~cm}, 35-50 \mathrm{~cm}, 51-65 \mathrm{~cm},>65 \mathrm{~cm}$ ). The size at the withers of sheltered dogs was measured by authorized shelter personal (veterinarian or shelter keeper) using a tape meter. Dogs were placed at the examination table or stood on the floor and their size at withers was taken. The effects of these independent variables on LOS as a dependent variable were analysed. For LOS, normality was checked using Kolmogorov-Smirnov test (Zar 1999). As data were not distributed normally, non-parametric methods were used for testing. First of all, a median for LOS was calculated for each level of the monitored independent variable in abandoned and lost dogs. The effects of the variables reason-for-admission and sex were analysed by two-tailed Mann-Whitney U test. The effects of age and size were analysed using Kruskal-Wallis ANOVA and subsequently by non-parametric multisample median test (Zar 1999) as post hoc test for pairwise comparisons. We also calculated the actual and relative frequencies of lost and abandoned dogs in selected categories according to sex, age, and size, and analysed the differences among these categories. Frequencies were compared on the basis of chi-square analysis of $2 \times 2$ contingency tables (Zar 1999). $P$ value $<0.05$ was considered as significant. To assess correlations in the study, Spearman rank correlation coefficients were calculated between LOS and age, and LOS and size of dogs.

\section{Results}

From 2010 to 2013, a total of 3,875 impounded dogs were housed by the three shelters analysed in this study. Of these, 1,614 dogs were subsequently reclaimed by their owners and 2,261 dogs were regarded as abandoned by their owners and offered for adoption.

We studied the sex, age, and size of dogs as important factors influencing LOS in shelters. Male dogs predominated in both the 'abandoned' and 'lost' groups. The numbers of each sex processed in this study and their LOS at the shelter are summarized in Table 1. Males accounted for $56 \%$ of abandoned dogs and $67.5 \%$ of lost dogs. The median LOS of all abandoned dogs was 23 days. Abandoned male dogs stayed in shelters significantly longer (median 27 days) compared to females (median 21 days). There was a significant difference $(P<0.001)$ between the median LOS of abandoned males and females. Lost male dogs stayed in the shelter for one day until being reclaimed by their original owners, 
unlike females, which were reclaimed by their owners on the day of admission to the shelter. However, when comparing sexes, there was no significant difference between LOS for lost dogs.

Table 1. Numbers and median lengths of stay (LOS) of males and females admitted to shelters.

\begin{tabular}{lcccrcr}
\hline & \multicolumn{3}{c}{ Abandoned dogs } & \multicolumn{3}{c}{ Reclaimed dogs } \\
\cline { 2 - 7 } Sex & \multicolumn{1}{c}{$(\mathrm{n})$} & $(\%)$ & LOS (days) & $(\mathrm{n})$ & $(\%)$ & LOS (days) \\
\hline Male & 1267 & 56.04 & 27 & 1089 & 67.47 & 1 \\
Female & 994 & 43.96 & 21 & 525 & 32.53 & 0 \\
\hline
\end{tabular}

Table 2 summarizes our findings on the effect of the age of abandoned and lost dogs on the frequency and length of their stays in shelters. Most abandoned dogs were aged up to 1 year $(48.9 \%)$, followed by dogs aged between one and three years $(26.5 \%)$, dogs aged between four and six years $(17.2 \%)$, and dogs aged between seven and nine years (5\%). Only $2.4 \%$ of abandoned dogs were aged over 9 years. Older abandoned dogs had significantly longer LOS in shelters before being adopted. The youngest abandoned dogs, i.e. dogs up to one year of age (puppies), had the shortest LOS in shelters (median 19 days), followed by dogs between one and three years of age (median 27 days), dogs between four and six years of age (median 34 days), dogs between seven and nine years of age (median 53.5 days), and dogs over 9 years of age (median 54 days). There were significant differences $(P<0.05)$ between these age groups and their total LOS in shelters. We also found a positive correlation between the age and LOS of dogs at shelters before being adopted $(\mathrm{r}=0.237, P<0.001, \mathrm{df}=2.259)$. Most lost sheltered dogs were in the $1-3$ years of age group $(32.2 \%)$, i.e. relatively young dogs. Lost dogs in the 4-6 years of age group formed the second largest category $(27.1 \%)$. Lost dogs over 9 years of age admitted to shelters formed the smallest group (9.4\%). There were no significant differences between the LOS of sheltered lost dogs according to age.

Table 2. Numbers and median lengths of stay (LOS) of five age categories of dogs admitted to shelters.

\begin{tabular}{lrrrrrr}
\hline \multirow{3}{*}{ Age (years) } & \multicolumn{3}{c}{ Abandoned dogs } & \multicolumn{3}{c}{ Reclaimed dogs } \\
\cline { 2 - 7 } & $(\mathrm{n})$ & $(\%)$ & LOS (days) & $(\mathrm{n})$ & $(\%)$ & LOS (days) \\
\hline$<1$ & 1106 & 48.92 & 19 & 319 & 19.76 & 0 \\
$1-3$ & 599 & 26.49 & 27 & 519 & 32.16 & 1 \\
$4-6$ & 389 & 17.20 & 34 & 437 & 27.08 & 1 \\
$7-9$ & 112 & 4.95 & 53.5 & 187 & 11.59 & 1 \\
$>9$ & 55 & 2.43 & 54 & 152 & 9.42 & 1 \\
\hline
\end{tabular}

Dog size was the last factor we examined. The numbers of both abandoned and lost dogs in size categories processed in this study and their LOS at shelters are summarized in Table 3. This table shows that the majority of abandoned dogs $(55.2 \%)$ in shelters were the smallest sized dogs, i.e. up to $35 \mathrm{~cm}$ at the withers. Significantly fewer dogs $(28.5 \%)$ were in the next largest size category $(35-50 \mathrm{~cm})$ and even fewer in the remaining groups. We found a positive correlation between the size of abandoned dogs and the LOS of these dogs in shelters before being adopted $(\mathrm{r}=0.087, P<0.001, \mathrm{df}=2.259)$. The category of giant dogs (dogs over $65 \mathrm{~cm}$ at the withers) spent demonstrably the shortest LOS in shelters (16 days) compared to all other size categories. However, during the studied period (2010-2013), there were only 7 abandoned dogs in this size category that were sheltered, 
which makes them highly unusual in Czech shelters. Dogs over $65 \mathrm{~cm}$ (giant dogs) were followed by the smallest group of abandoned dogs (up to $35 \mathrm{~cm}$ ), with a median LOS of only 21 days in shelters. Dogs in the $35-50 \mathrm{~cm}$ category spent 29 days in shelters, and dogs in the 51-65 cm category spent 30 days in shelters. Significant differences $(P<0.001)$ in the LOS of abandoned dogs were found among all size categories of abandoned dogs. The overall numbers of lost dogs in different size categories were very similar if we exclude the category of giant dogs (over $65 \mathrm{~cm}$ ), which, during the studied period, were represented by only 10 individuals $(0.6 \%)$. The LOS of lost dogs according to size did not differ and there were no significant differences between size categories.

Table 3. Numbers and median lengths of stay (LOS) of four size categories of dogs admitted to shelters.

\begin{tabular}{lrccccc}
\hline \multirow{2}{*}{ Size $(\mathrm{cm})$} & \multicolumn{3}{c}{ Abandoned dogs } & \multicolumn{3}{c}{ Reclaimed dogs } \\
\cline { 2 - 7 } & \multicolumn{1}{c}{$(\mathrm{n})$} & $(\%)$ & LOS (days) & $(\mathrm{n})$ & $(\%)$ & LOS (days) \\
\hline$<35$ & 1248 & 55.20 & 21 & 570 & 35.32 & 1 \\
$35-50$ & 644 & 28.48 & 29 & 507 & 31.41 & 1 \\
$51-65$ & 362 & 16.01 & 30 & 527 & 32.65 & 0 \\
$>65$ & 7 & 0.31 & 16 & 10 & 0.62 & 0 \\
\hline
\end{tabular}

\section{Discussion}

Factors affecting adoptions of dogs from shelters can be broadly divided into factors related to the dogs themselves, factors related to potential adopters, and a variety of other factors, such as the time of year, legal issues, and factors associated with shelter management etc. Factors linked to dogs include sex, age, size, coat colour, breed, behaviour, temperament, etc. One recent study showed that certain factors such as age and size have a particular value for the adoption potential (Siettou et al. 2014). The appearance of sheltered dogs is one of the most influential factors. When asked about the most important factors in choosing a particular dog, adopters most frequently cited appearance, behaviour with people, and age (Weis s et al. 2012). In particular, keepers in shelters know that the sex, age, and size of dogs are important factors for potential adopters when selecting the right dog and usually, such information is made available to potential adopters.

Total numbers of abandoned animals, i.e. those not reclaimed by their original owners, predominated in all three surveyed shelters. These high numbers of abandoned dogs compared to lost dogs may be an indicator of the current trend that people often get rid of their dogs after losing interest in them.

The median time until adoption for abandoned dogs in Czech shelters was 23 days, which is considerably shorter than that found in other studies (Němcová and Novák 2003; Normando et al. 2006; Diesel et al. 2007; Brown et al. 2013). According to our results, females have demonstrably shorter LOS in shelters compared to male dogs. This finding is supported by several other studies (Patronek et al. 1995; Lepper et al. 2002; Clevenger and Kass 2003; Marston et al. 2004; Mondelli et al. 2004; Diesel et al. 2007). These studies also showed that females are more likely to be adopted. According to Diesel et al. (2007), females are more frequently adopted than males, because female dogs generally have a calmer nature. It is also possible that the shorter LOS in females is the result of their lower numbers in shelters; in our study, $42.96 \%$ of abandoned dogs were females. Also, male dogs tend to stray more than females due to their natural sexual behaviour and male dogs can also show more aggression than females, which makes them less attractive for potential adopters (Wells and Hepper 2000). Interestingly, a Czech study from 11 years ago (Němcová and Novák 2003) did not reveal any differences in 
the adoption potential based on the sex of abandoned dogs. However, many changes may have occurred in the Czech society over the last decade with respect to dog breeding and the availability of general information about abandoned animals and their characteristics. Therefore, our results or the results of other recent studies may differ from those of Němcová and Novák (2003). One recent U.S. study also found no significant differences in the LOS of sheltered dogs based on sex (Brown et al. 2013). In contrast, on the basis of a survey in Brazil (Soto et al. 2005), it was found that potential adopters preferred male dogs to bitches. According to these authors, the reasons were that female dogs can have unwanted litters, and that males require less care and attention. We did not monitor whether dogs admitted to shelters were neutered or not; thus, we have no data on whether potential adopters prefer animals sexually intact or neutered. However, some foreign studies reported that neutered individuals are more adoptable than sexually intact individuals (Lepper et al. 2002; Clevenger and Kass 2003).

We also examined the age of dogs as one of the factors influencing choices among potential adopters and the numbers of dogs admitted to the shelter. According to our results it can be assumed that younger dogs tend to stray more and run away from owners more often than puppies or older dogs. Our results show that the youngest abandoned dogs (i.e. puppies up to 1 year of age) have the shortest LOS in shelters. This finding is confirmed by one Czech study (Němcová and Novák 2003) and also by many other studies (Patronek et al. 1995; Hart et al. 1998; Lepper et al. 2002; Previde et al. 2003; Brown et al. 2013). Brown et al. (2013) also found that LOS did not depend on other factors such as the size of the puppy, sex, or breed group. According to our results, the median LOS for abandoned dogs up to 1 year of age were almost three times shorter than for dogs over 6 years of age and over 9 years of age. Furthermore, it was shown that the oldest age group (over 9 years) spent the longest LOS in the surveyed shelters. The finding that the oldest dogs remain in shelters significantly longer compared to younger dogs has been reported by other studies (Lepper et al. 2002; Normando et al. 2006) and the conclusions of these studies are consistent with ours. Older dogs in shelters can suffer from a lack of interest from potential adopters because of the increased risk of such dogs having health problems and the consequent likelihood of higher health care costs (Clancy and Rowan 2003).

Our study also examined the effect of the size of abandoned and lost dogs on the frequency and length of stay in shelters. The surveyed shelters admit mostly abandoned dogs up to $35 \mathrm{~cm}$ in height, i.e. small sized dogs. Large-sized dogs can generally spend more time outdoors and may not require as much attention as small sized dogs, which are usually kept indoors. Dogs kept indoors may be a problem for householders (Salman 1998). With respect to abandoned dogs, giant dogs (dogs over $65 \mathrm{~cm}$ ) demonstrably spent the shortest time in shelters. Giant dogs are easily recognizable due to their imposing appearance and, in shelters, relatively unusual, which might make them more desirable than dogs of other groups (Brown et al. 2013). It is important to note that even though there were more abandoned small-sized dogs in shelters, they were still among the group of dogs with the shortest LOS. In our study, medium-sized and large-sized dogs had demonstrably the longest length of stays in shelters. This finding is confirmed by Brown et al. (2013), who found that medium-sized dogs had the longest length of stay in shelters, while extremely small dogs and puppies stayed in shelters the shortest time of all. Posage et al. (1998) also showed that the small size of dogs was a significant indicator of successful adoption. Similarly, in a study conducted in the UK, Siettou et al. (2014) found that potential adopters preferred smaller dogs to larger ones. Size may be an important factor for adopters of dogs, but there may be housing restrictions which limit the choices of potential adopters with respect to large-sized dogs (Moorhead 1998; Shore et al. 2003). Large dogs may also be less adoptable because of concerns of potential owners regarding the safety of young children (Chun et al. 1982). There may also be concerns about the safety of other 
pets (Clancy and Rowan 2003). In the Czech context, however, potential adopters could prefer small-sized dogs simply because they are less costly to keep.

Nevertheless, it is also important to note that all the three surveyed shelters question potential adopters about the conditions in which the animal would live and how much time the owners would have to devote to the dog etc. It is therefore logical to conclude that the final decision about whether an animal will be adopted or not is made by the shelter staff.

In conclusion, it was found that the sex, age, and size of sheltered abandoned dogs have a significant effect on both the LOS in Czech dog shelters and the likelihood of a dog being adopted by a new owner. Abandoned male dogs in shelters remained demonstrably longer than abandoned females. The oldest abandoned dogs, i.e. over 9 years of age, and large-sized dogs measuring $51-65 \mathrm{~cm}$ at the withers had the longest LOS. In contrast, the youngest abandoned dogs, i.e. up to 1 year of age, giant dogs over $65 \mathrm{~cm}$ at the withers, and small-sized dogs up to $35 \mathrm{~cm}$ at the withers had the shortest LOS in Czech shelters. Recognizing the preferences of potential adopters and the likelihood of individual dogs being adopted are crucial factors in the establishment of effective procedures for rehoming shelter dogs.

\section{References}

Brown WP, Davidson JP, Zuefle ME 2013: Effects of phenotypic characteristics on the length of stay of dogs at two no kill animal shelters. J Appl Anim Welf Sci 16: 2-18

Clancy E, Rowan A 2003: Companion animal demographics in the United States: A historical perspective. In: State of the Animals II. Washington: Humane Society of the United States 9-26

Clevenger J, Kass P 2003: Determinants of adoption and euthanasia of shelter dogs spayed or neutered in the University of California Veterinary Student Surgery Program compared to other shelter dogs. J Vet Med Educ 30: $372-378$

Diesel G, Smith H, Pfeiffer D 2007: Factors affecting time to adoption of dogs re-homed by a charity in the UK. Anim Welf 16: 353-360

Hart L, Takayanagi T, Yamaguchi H 1998: Dogs and cats in animal shelters in Japan. Anthrozoös 11: 157-163

Chun Y, Berkelharner J, Herold T 1982: Dog bites in children less than 4 years old. Pediatrics 69: 119-120

Lepper M, Kass P, Hart L 2002: Prediction of adoption versus euthanasia among dogs and cats in a California animal shelter. J Appl Anim Welf Sci 5: 29-42

Marston L, Bennett P, Coleman G 2004: What happens to shelter dogs? An analysis of data for 1 year from three Australian shelters. J Appl Anim Welf Sci 7: 27-47

Marston L, Bennett P, Coleman G 2005: What Happens to Shelter Dogs? Part 2. Comparing Three Melbourne Welfare Shelters for Nonhuman Animals. J Appl Anim Welf Sci 8: 25-45

Moorhead T 1998: A practical approach to landlord/tenant obligations under the federal fair housing act and the Michigan handicappers' civil rights act. Michigan Real Property 25: 111

Mondelli F, Previde E, Verga M, Levi D, Magistrelli S, Valsecchi P 2004: The bond that never developed: Adoption and relinquishment of dogs in a rescue shelter. J Appl Anim Welf Sci 7: 253-266

Němcová D, Novák P 2003: Adoption of dogs in the Czech Republic. Acta Vet Brno 72: 421-427

Normando S, Stefanini C, Meers L, Adamelli S, Coultis D, Bono G 2006: Some factors influencing adoption of sheltered dogs. Anthrozoös 19: 211-224

Notaro SJ 2004: Disposition of shelter companion animals from nonhuman animal control officers, citizen finders, and relinquished by caregivers. J Appl Anim Welf Sci 7: 181-188

Patronek G, Glickman L, Moyer M 1995: Population dynamics and the risk of euthanasia for dogs in an animal shelter. Anthrozoös 8: 31-43

Posage J, Bartlett P, Thomas D 1998: Determining factors for successful adoption of dogs from an animal shelter. J Am Vet Med Assoc 213: 478-482

Previde E, Custance M, Spiezio C, Sabatini F 2003: Is the dog-human relationship an attachment bond? An observational study using Ainsworth's strange situation. Behaviour 140: 225-254

Protopopova A, Gilmour AJ, Weiss RH, Shen JY, and Wynne CDL 2012 The effects of social training and other factors on adoption success of shelter dogs. Applied Animal Behaviour Science 142: 61-68

Salman MD, New JC, Scarlett JM, Kass PH, Ruch-Gallie R, Hetts S 1998: Human and animal factors related to the relinquishment of dogs and cats in 12 selected animal shelters in the United States. J Appl Anim Welf Sci 1: $207-226$

Shore E, Petersen C, Douglas K 2003: Moving as a reason for pet relinquishment: A closer look. J Appl Anim Welf Sci, 6: 39-52

Siettou C, Fraser IM, Fraser RW 2014: Investigating Some of the Factors That Influence "Consumer" Choice When Adopting a Shelter Dog in the United Kingdom. J Appl Anim Welf Sci 17: 136-147 
Soto F, Ferreira F, Pinheiro S, Nogari F, Risseto M, de Souza O, Amaku M 2005: Adoption of shelter dogs in a Brazilian community: Assessing the caretaker profile. J Appl Anim Welf Sci 8: 105-116

Voslářová E, Žák J, Večerek V, Bedáňová I 2015: Breed characteristics of abandoned and lost dogs in the czech republic. J Appl Anim Welf Sci 18: 332-342

Weiss E, Miller K, Mohan-Gibbons H, Vela C 2012: Why did you choose this pet? Adopters and pet selection preferences in five animal shelters in the United States. Animals 2: 144-159

Wells DL, Hepper PG 2000: Prevalence of behaviour problems reported by owners of dogs purchased from an animal rescue shelter. Appl Anim Behav Sci 69: 55-65

Wells DL, Graham L, and Hepper PG 2002 The influence of length of time in a rescue shelter on the behaviour of kennelled dogs. Animal Welfare 11: 317-325

Zar JH 1999: Biostatistical Analysis. Upper Saddle River: Prentice Hall 